\section{NICE says doctors could help 165000 more smokers to quit}

Balaji Ravichandran BMJ

If GPs encouraged more of their patients to stop smoking by opportunistic advice and referring some patients to intensive support clinics, the number of smokers quitting each year in England would increase by 165000 , the National Institute for Health and Clinical Excellence (NICE) said last week.

NICE calculates that if doctors took such measures the proportion of England's 10 million smokers giving up for more than a year would increase from $2.7 \%$ to $4.3 \%$.

The institute published the figures when it launched its first public health guidance, covering smoking and physical activity. become more active."
Andrea Sutcliffe, deputy chief executive of NICE, said, "We expect this guidance will support practitioners, patients, and the public by making it clear that improving health depends on prevention, not just cure

[The guidance] considers the effectiveness of specific ways to help people stop smoking and

The guidance on smoking cessation recommends "brief interventions" in primary care and other settings, involving opportunistic advice, discussion, negotiation, and encouragement. Such interventions would typically last for 5 to 10 minutes.

In certain cases patients should be offered pharmacotherapy, behavioural support, or referral to more intensive support, such as the NHS's smoking cessation services, the guidance says.

Dagmar Zeuner, a consultant in public health and a member of the independent Public Health Interventions Advisory Committee, which formulated the recommendations, said, "The guidance message is clear: if you're a smoker, the most important thing you can do for your health is to stop smoking, and a brief chat with your GP can help you on the path to quitting."

The NICE guidance recommends four "commonly used" methods to increase physical activity: brief interventions in primary care, referral to exercise schemes, pedometers, and organised walks or rides. The guidance recommends sensitivity to individual needs and care- ful scrutiny to avoid inappropriateness during consultations.

Matt Kearney, a GP and another member of the Public Health Interventions Advisory Committee, said: "If the guidance is implemented as planned, the number of smokers who quit will increase by 165000 per year This is a very cost effective way to prevent mortality due to complications of cigarette smoking."

$\mathrm{He}$ added, "There is a clear link between physical inactivity and ill health. Regular physical activity plays a role in reducing the risk of diabetes and colon cancer, and active people have a $50 \%$ lower risk of coronary heart disease."

Implementation of the guidance will be closely monitored by the Healthcare Commission, NICE said.

\section{The guidance is available at} www.nice.org.uk.

\section{Meta-analysis challenges benefits of moderate drinking}

Janice Hopkins Tanne New York

Imprecision in the classification of "abstainers" invalidates the results of studies showing the benefits of moderate drinking, a meta-analysis says.

The meta-analysis of 54 published studies from the past 38 years says that classing people who had reduced their drinking or stopped as abstainers invalidates the results of most studies showing the benefits of moderate drinking.

"Cardiac protection afforded by alcohol may have been overestimated," reports the study, which was published online ahead of print publication in the May 2006 issue of Addiction Research and Theory (doi: 10.1080/ 16983).

The researchers, from the University of California at San Francisco and the University of British Columbia, said that most of the 54 prospective studies were flawed as they included as abstainers people who had reduced or stopped drinking, which people often do because of ageing or ill health. Abstainers thus seemed to be less healthy than light drinkers and had a higher risk of death.

After considering 57 variables the researchers found just seven of the studies to be free of error and to include long term abstainers. Analysis of these studies showed no reduction in mortality among moderate drinkers in comparison with abstainers. Only studies containing the "abstainer error" showed protection against death with moderate drinking

Kaye Fillmore, of the University of California's School of Nursing, a coauthor, said, "The study reopens the debate about the protective effect of alcohol for coronary heart disease."

She said that most studies contained a systematic or misclassification error by including as "abstainers" people who had stopped or reduced drinking because of age or illness. People reduce their consumption of alcohol as they age, become more frail, or take drugs that may interact with alcohol. Including these people in the category of abstainers raised the risk of mortality among true abstainers.

Dr Fillimore said, "This was interpreted to mean that abstinence from drinking was associated with increasing mortality among abstainers, when in fact it was probably because these people were ill and frail [and had

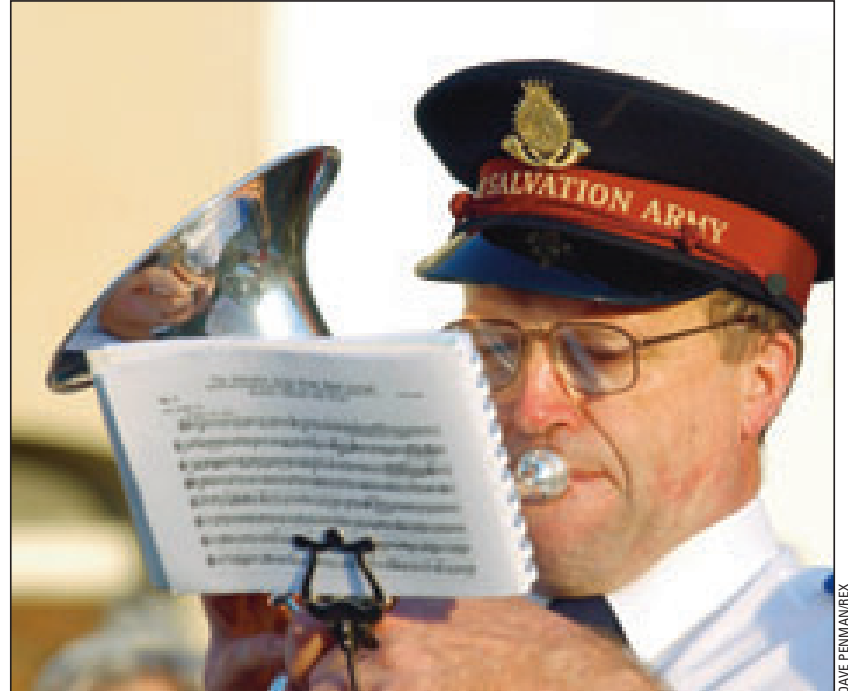

The blessings of abstinence: was the Salvation Army right all along?

reduced their drinking]."

Repetition of this error in studies contributed to the idea that alcohol had a protective effect on moderate users, she said.

She said that a British researcher, Professor Gerry Shaper of the Royal Free Hospital and University College Medical School, had suggested in 1988 that many studies were flawed because people who had reduced or stopped drinking because of age or illness were put into the abstainer category, thus elevating the risk for abstainers (Lancet 1988; 2:12671273)
The studies that the researchers reviewed "varied enormously in what they controlled for," she said. The definitions of light, heavy, and moderate drinking varied, for instance. The definition of a "standard drink" varied. The period of abstinence varied from as much as one year to much shorter periods. Some studies didn't include people who drank less than once a week, others didn't include participants history of smoking. Yet another group did not differentiate between the use of wine, beer, or spirits. 